\title{
Performance Analysis of Opportunistic Relaying
} and Opportunistic Hybrid Incremental Relaying

\section{Over Fading Channels}

\author{
Hafiz Yasar Lateef, Vladimir Dyo, Ben Allen \\ Centre for Wireless Research \\ University of Bedfordshire \\ Park Square, Luton, LU1 3JU, UK \\ \{lateef.hafiz, vladimir.dyo, ben.allen\}@beds.ac.uk
}

\begin{abstract}
In this paper, we develop and present a comprehensive analysis of two opportunistic cooperative relaying schemes for LTE-Advanced networks operating over Generalized- $K$ and Nakagami- $m$ fading channels. We present and compare the performance of opportunistic relaying (OR) and opportunistic hybrid automatic repeat request (Hybrid-ARQ) incremental relaying (OHIR). We analyse performance in terms of the average symbol error rate (ASER) for both conventional OR and OHIR LTE-Advanced networks with the radio channel modelled as composite Generalized- $K$ fading (encompassing both fading and shadowing) and Nakagami- $m$ fading channels. We also analyse the outage probability for OR operating over these channels. Both the theoretical analysis and simulations confirm that for conventional OR LTE-Advanced networks operating over composite Generalized- $K$ fading channels, a diversity order of $k(N+1)$ is achieved when shadowing is more severe than fading, and a diversity order of $m(N+1)$ is achieved when fading is more severe than shadowing (where $k$ and $m$ represent the Generalized- $K$ distribution shape parameters and $N$ represents the number of candidate relays for the OR selection). The simulation results confirm the accuracy of the analytical expressions developed in this paper. It is evident from the theoretical analysis and simulations that, for a similar quality of service as that for OR, OHIR not only reduces the amount of required radio resources but also maintains the full diversity order.
\end{abstract}

\section{Index Terms}

Cooperative Relaying, Hybrid-ARQ, Opportunistic Relaying, amplify-and-forward, Generalized- $K$ fading, LTEAdvanced, Heterogeneous Networks. 


\section{INTRODUCTION}

OOPERATIVE relaying is one of the most promising techniques for extended coverage and increased cellular capacity in future 4G LTE-Advanced Heterogeneous Networks (HETNETS) [1],

[2]. The relaying terminals may be either mobile devices or fixed terminals that extend the coverage while avoiding the infrastructure and operational cost that the deployment of base stations (Evolved Node B or eNB) entails [2]. Depending upon the ability of the relaying terminals to manage their radio resources, relays can be divided into 2 categories according to the 3GPP specifications, i.e., Type- 1 relay or Type- 2 relay [1]. Cooperative relaying is robust against small-scale fading and offers resilience against attenuation due to path loss and shadowing. The most common cooperative relaying protocols were introduced in [3] and [4].

Scaling cooperation to accommodate multiple relays while maintaining high spectral efficiency and simplifying radio resource management for HETNETS is of particular interest to researchers and 3GPP standards bodies. One possible approach is the use of distributed space-time coding among participating nodes [5]. However, distributed space-time code design is difficult to implement in practice owing to the ad-hoc nature of the cooperative links and the varying number of relays, since coordination among cooperative relays is required prior to their use such that a suitable space-time coding scheme may be selected.

The opportunistic relaying protocol proposed in [6] and [7] is one approach to minimise the required coordination overhead and simultaneously to realise the potential benefits of cooperation between multiple relays. Opportunistic relaying protocols select the best relay between the source and destination based on a maximum signal-to-noise ratio policy, and require minimal signalling overhead [6]. This opportunistic relaying method requires no knowledge of the topology, simplifies the required space-time coding and limits the number of orthogonal transmissions to two. The advantage of the conventional OR scheme comes at the cost of spectral efficiency because of transmission from relays in orthogonal channels (e.g. TDMA). Therefore, the attained spectral efficiency through this scheme is proportional to $(1 / N+1)$, where $N$ is the number of relays, and it is apparent that the spectral efficiency reduces as $N$ increases. This is not the optimal means of using scarce radio resources such as channel time slots. In order to increase spectral efficiency, a number of cooperative relaying schemes have recently been proposed.

Among the cooperative relaying schemes, incremental relaying transmission protocols, prescribing cooperation only upon erroneous decoding by the destination, emerged as promising techniques to achieve 
higher spectral efficiency [8]. This approach is an application of the Hybrid-ARQ incremental redundancy principle to a cooperative relaying environment [8]. In contrast, retransmitted packets in the cooperative Hybrid-ARQ scheme do not come from the original source node but could instead be retransmitted by the relays that overhear the source transmission [8]. Cooperative Hybrid-ARQ incremental redundancy protocol can be implemented by exploiting a limited feedback from the destination terminal, (e.g., a single bit indicating the success or failure of the direct transmission) [9]. More specifically, if the source-todestination signal-to-noise ratio (SNR) is not sufficiently high for successful direct transmission, then the feedback from the destination requests the relay to resend the data that it received. The authors in [10] proposed a new approach called incremental-best-relay technique or opportunistic Hybrid-ARQ incremental relay (OHIR). In OHIR networks, retransmission of the source signal, when the destination provides a negative acknowledgment via feedback, is done by the best relay only (among the $N$ candidate relays) [9], [10].

Wireless signal propagation may be characterised by the joint effect of two independent random processes, namely, multipath fading and shadowing. In order to predict accurately the performance of a wireless network, composite channel models such as Rayleigh-lognormal, Rician-lognormal, and Nakagamilognormal distributions have been proposed, which encompass multipath-fading and shadowing. The Generalized- $K$ channel model has attracted considerable attention recently [11]: it is a general channel model that can describe the combined effect of slow and fast fading of the signal in a single expression [12]. This provides a convenient way of analysing link performance over a range of slow and fast fading parameters in a single expression.

The average symbol error rate (ASER) and the ergodic capacity of conventional OR networks operating over Nakagami- $m$ fading radio channels have been presented in [13]. Recently, the authors in [9] presented the ASER, outage analysis and the average channel capacity of OHIR networks operating over Rayleigh radio channels. Yadav et al [16] derived the upper and lower bounds on the outage probability, and obtained approximate expressions for average symbol error probability for amplify-and-forward (AF) relaying scheme over cascaded Generalized-K fading channels. Bissias et al [17] analysed the performance of dual-hop relaying with best relay selection over Generalized-K fading channels and obtained an upper bound on end-to-end SNR to derive approximate expressions for the average symbol error and outage probabilities. Moreover, Alouini et al., presented the average bit error rate and the outage analysis of OHIR network with adaptive modulation over Rayleigh radio channels [10]. 
Whilst these contributions are significant in their own right, their description in the literature has some limitations, which are:

- the conventional OR network considered in [13], [15],[17] does not cover the case of a spectrally efficient OHIR network;

- the analysis in [13] and [15] does not consider the diversity order of OR networks and the impact of system parameters on the error performance;

- the performance analysis of OHIR networks in [9] and [10] is restricted to Rayleigh radio channels and does not cover the more realistic composite multipath fading channels, together with shadowing scenarios such as Generalized- $K$ and Nakagami- $m$ radio channels, while the performance analysis in [9] and [10] does not provide information relating to the diversity and coding gains; and

- the outage probability and the ASER expressions in [10], [13], [15] result in multi-level summations and the application of numerical techniques to solve the equations, which increase the computational complexity. The analysis in [16] considers an AF relaying scheme over cascaded Generalized- $K$ fading channels, but does not apply to schemes with opportunistic relay selection.

Most of the previous work for scaling cooperation to multiple relays focuses on conventional OR networks operating over multipath fading channels. This is not spectrally efficient owing to unconditional retransmission from the relay nodes, irrespective of the feedback from the destination terminal. On the other hand, the performance analysis of conventional OR and spectrally efficient OHIR networks operating over realistic multipath fading channels, together with shadowing scenarios (e.g. Generalized- $K$ and Nakagami- $m$ radio channels) have never been addressed before to the best of our knowledge. Moreover, the diversity gain, coding gain and the effect of system parameters of OHIR networks operating over realistic multipath fading channels, together with shadowing, have never been taken into account either. References [19] and [23] investigate the performance of cooperative multi-hop relay networks with the radio channel modelled as a Generalized- $K$ channel, but their analysis was limited to networks where a signal is relayed unconditionally through a fixed chain of relay nodes, and cannot be applied or extended to OR or OHIR networks.

To address these limitations we now present a comprehensive performance analysis of both conventional OR and OHIR networks operating over Generalized- $K$ and Nakagami- $m$ radio channels in terms of ASER. In addition, we develop the analysis of the outage probability for OR operating over these channels. More specifically, we first derive the new closed-form expressions for the Cumulative Distribution Function 
(CDF) and the Probability Density Function (PDF) of the end-to-end SNR of conventional OR and OHIR networks operating over Generalized- $K$ and Nakagami- $m$ radio channels. Our expressions are approximations, which stem from approximations necesarily used in the relating literature in order to yield tractable closed-form expressions. Thus, the search for tractable closed-form exact expressions remains an open research question. We then utilise these results to derive closed-form expressions for the ASER of both conventional OR and OHIR networks and the outage probability for OR networks. We verify the analytical results against those obtained by Monte Carlo simulation. The performance metric is ASER which has been determined in this paper for OR and OHIR in terms of SNR and N, where $\mathrm{N}$ in the number of candidate relays. In addition, the outage probability, $P_{\text {out }}$, has been determined for OR in terms of SNR and N. The expressions that have resulted from our analysis are 5, 17, 29, 30, 33 and 45 respectively. Spectral efficiency is not explicitly explored, but could subsequently be incorporated into future work. The inherently higher spectral efficiency of OHIR compared to OR is, however, noted. The utility of the resulting expressions are that they can be used to validate simulation platforms as well as to provide a means of exploring performance without the need to develop a full simulator. Thus, they provide a theoretical basis that underpins simulation results. The expressions have been validated against results obtained from a very well established simulator, as detailed later. More specifically, the expressions identified above allow ASER and $P_{\text {out }}$ to be determined for the defined scenarios.

The rest of this paper is organised as follows. In section II, we describe the system and channel model used throughout this paper. Closed-form expressions for the ASER of amplify-and-forward (AF) conventional OR networks operating over Generalized- $K$ and Nakagami- $m$ radio channels are presented in section III. Section IV give a comprehensive performance analysis of OHIR networks operating over Generalized- $K$ and Nakagami- $m$ radio channels. Numerical and simulation results for the ASER and the outage probabilities are presented in section V. Finally, section VI draws conclusions based upon the analysis in the previous sections.

\section{System And Channel Model}

We consider opportunistic relaying where an Evolved Node B (eNB) is communicating with the user equipment (UE) ${ }^{1}$ through the best relay $\left(R_{b}\right)$ selected among the $N$ candidate relays $R_{i}, i \in\{1, \cdots, N\}$, as shown in Figure 1.

\footnotetext{
${ }^{1}$ Throughout this paper Evolved Node B (eNB) is represented as source node $(S)$ and UE is also termed as destination $(D)$.
} 


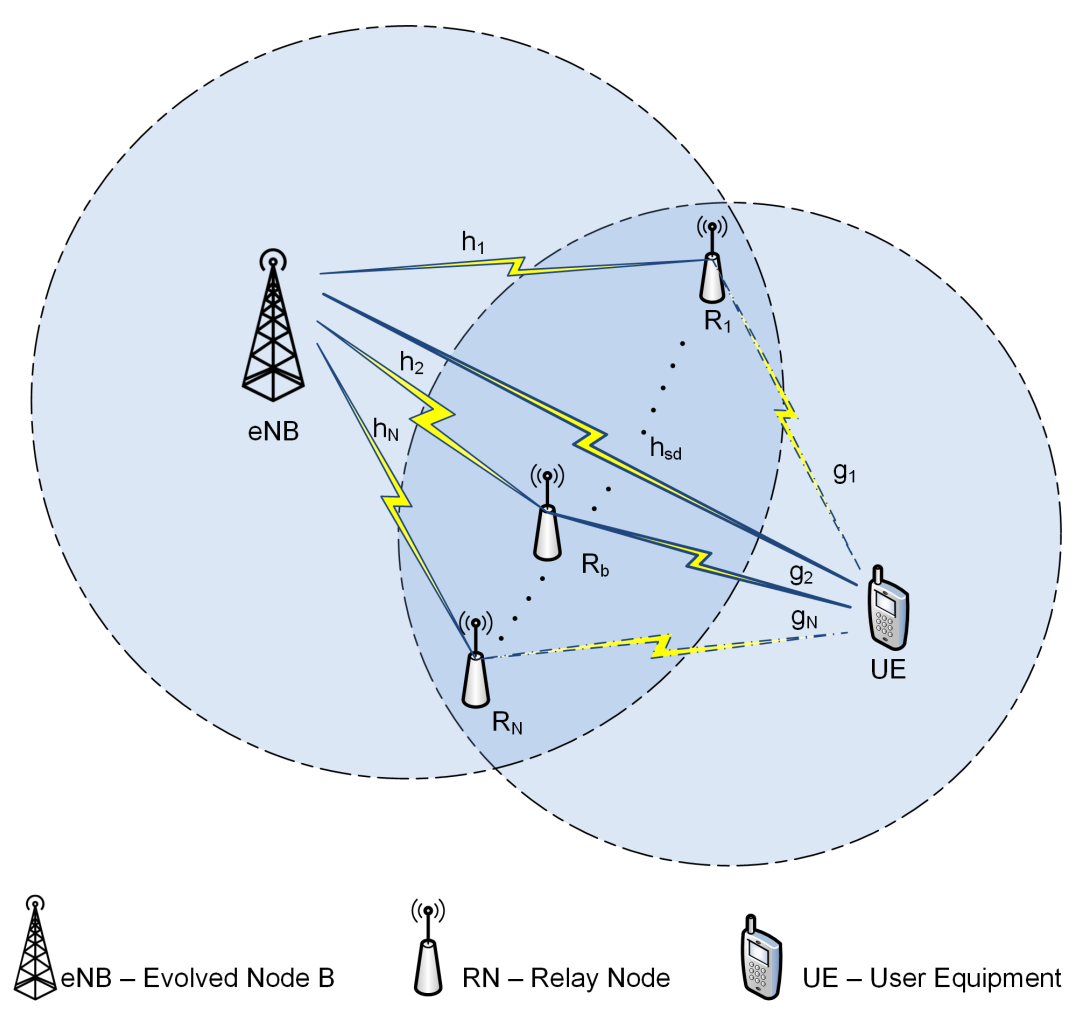

Figure 1. Illustration of an opportunistic cooperative relaying LTE-Advanced Heterogeneous Network. The best relay (Rb) amplifies and forwards the signal to the destination, which combines it with the direct signal from the source.

The channel coefficients $h_{i}$ (between the source and relay $R_{i}$ ), $g_{i}$ (between relay $R_{i}$ and the destination) and $h_{s d}$ (between the source and the destination) are independent and non-identical Generalized- $K$ or Nakagami- $m$ fading coefficients. Each node operates in half-duplex mode. In the first time slot, the source transmits the signal to the destination and relays $\left(R_{i}\right)$. In conventional $\mathrm{OR}$, the best relay amplifies the signal received from the source and forwards it to the destination in the second time slot, whereas in OHIR, the destination first decides whether opportunistic relaying is required or not, and then for the case of insufficient signal quality the best relay amplifies the signal received from the source and forwards it to the destination in the second time slot. Note that (a) $N_{0, i}=N_{0}$ is the single-sided power spectral density of the Additive White Gaussian Noise (AWGN) at the input of the relay $R_{i}$; (b) $G_{i}=\sqrt{E_{s} /\left(\left|h_{i}\right|^{2} E_{s}+N_{0, i}\right)}$ denotes the gain of the relay $R_{i}$. This definition of the gain may result in large values for low SNR conditions. To keep our analysis general and to understand the performance of the system, we assumed an ideal linear amplifier but acknowledge that in a practical implementation of such a scheme the gain of the amplifier would be limited; (c) $E_{s}$ is the average radiated energy per symbol; (d) $\gamma_{s r_{i}}=\left|h_{i}\right|^{2} E_{s} / N_{0}$ is the instantaneous SNR between the source and the relay $R_{i}$; (e) $\gamma_{r d_{i}}=\left|g_{i}\right|^{2} E_{s} / N_{0}$ is the SNR between the relay $R_{i}$ and the destination; (f) $\gamma_{s d}=\left|h_{s d}\right|^{2} E_{s} / N_{0}$ represents the SNR of the direct path between $S$ 
and $D ;(\mathrm{g}) \bar{\gamma}_{s r_{i}}=E\left\{\left|h_{i}\right|^{2}\right\} E_{s} / N_{0}$ is the average SNR between the source and the relay $R_{i}$ (where $E\{$. denotes the statistical expectation operator); (h) $\bar{\gamma}_{r d_{i}}=E\left\{\left|g_{i}\right|^{2}\right\} E_{s} / N_{0}$ is the average SNR between the relay $R_{i}$ and destination (i) $\bar{\gamma}_{s d}=E\left\{\left|h_{s d}\right|^{2}\right\} E_{s} / N_{0}$ denotes the average SNR between $S$ and $D$. For Generalized- $K$ radio channels, the PDF $P_{h_{y}}(\vartheta)$ of the fading amplitude $h_{y}$ is given as [12]:

$$
P_{h_{y}}(\vartheta)=\frac{4 m_{y}^{\left(\sigma_{y}+1\right) / 2} \vartheta^{\sigma_{y}}}{\Gamma\left(m_{y}\right) \Gamma\left(k_{y}\right) \Upsilon_{y}^{\left(\sigma_{y}+1\right) / 2}} K_{\alpha_{y}}\left[2 \sqrt{\frac{m_{y}}{\Upsilon_{y}}} \vartheta\right]
$$

where $y=\left\{s r_{i}, r d_{i}, s d\right\}, \alpha_{y}=k_{y}-m_{y}, \sigma_{y}=k_{y}+m_{y}-1, \Upsilon_{y}=E\left\{\left|h_{y}\right|^{2}\right\} / k_{y}, \Gamma($.$) is the Gamma function$ [15, eq. (6.1.1)], $k_{y}, m_{y}$ are the distribution shape parameters and $K_{\alpha_{y}}($.$) is the modified Bessel function of$ order $\alpha_{y}$ [15, eq. (9.6.23)]. For Generalized- $K$ radio channels [12] , the PDF $P_{\gamma_{y}}(\rho)$ of the instantaneous SNR $\gamma_{y}$ is expressed as follows:

$$
P_{\gamma_{y}}(\rho)=\frac{2 \Xi_{y}^{\left(\sigma_{y}+1\right) / 2} \rho^{\left(\sigma_{y}-1\right) / 2}}{\Gamma\left(m_{y}\right) \Gamma\left(k_{y}\right)} K_{\alpha_{y}}\left[2 \sqrt{\Xi_{y} \rho}\right]
$$

where $\Xi_{y}=\left(k_{y} m_{y} / \bar{\gamma}_{y}\right)$. By considering channel state information (CSI)-assisted relays [19], the best relay, $R_{b}$, is pro-actively (before the source transmission) selected from the $N$ candidate relays, $R_{i}$, according to the opportunistic protocol [6], then by applying Maximum Ratio Combining (MRC) diversity at the destination, the SNR at the destination, $\gamma_{e n d}$, can be written as [9]:

$$
\gamma_{e n d}=\gamma_{s d}+\max _{i \in\{1, \ldots, N\}}\left(\gamma_{i}\right)
$$

Expression (3) is obtained by defining the gain of the $i^{t h}$ relay $\left(R_{i}\right)$ as $G_{i}=\sqrt{E_{s} /\left(\left|h_{i}\right|^{2} E_{s}+N_{0, i}\right)}$ and $\gamma_{i}$ is the tight upper bound of the dual-hop SNR from the $S-R_{i}-D$ combination, i.e. $\gamma_{S \rightarrow R_{i} \rightarrow D}$, which is defined in [9] as $\gamma_{S \rightarrow R_{i} \rightarrow D} \leq \gamma_{i}=\min \left(\gamma_{s r_{i}}, \gamma_{r d_{i}}\right)$.

\section{Performance Analysis of Conventional OR Networks}

\section{A. Average Symbol Error Rate}

The average symbol error rate probability, $\bar{P}_{s e}$, is a useful measure for evaluating the performance of wireless communication systems, and for a conventional OR network it may be evaluated for M-PSK, M-FSK and M-QAM modulation schemes as follows [13][14]:

$$
\bar{P}_{s e}=\int_{0}^{\infty} P_{s e}(e \mid \chi) P_{\gamma_{e n d}}(\chi) d \chi
$$


where $P_{s e}(e \mid \chi)$ is the conditional symbol error rate for AWGN and $P \gamma_{e n d}(\chi)$ is a PDF of SNR at the destination. Now, $P_{s e}(e \mid \chi)$ can be written as $P_{s e}(e \mid \chi)=A \operatorname{erfc}(\sqrt{C \chi})$, where $\operatorname{erfc}(\mathrm{x})=\frac{2}{\sqrt{\pi}} \int_{x}^{\infty} e^{-u^{2}} d u$ is the complementary error function [18, eq. (7.1.2)], and $A$ and $C$ are constants depending upon the type of modulation scheme used, where the values of $A$ and $C$ are detailed in Table 1 of reference [20] for a range of spectrally and power efficient digital modulation schemes.

In this section, we present the comprehensive ASER analysis of conventional OR networks where the radio channels are modelled as Generalized- $K$ and Nakagami- $m$ functions. In particular, we will consider an analysis for medium to high SNR operating scenarios, which allows us to gain insights relating to the impact which parameters such as the Generalized- $K$ fading parameters $m, k$ and the number of candidate relays for the OR selection $(N)$ have on the system performance, and which enable the diversity gain and coding gain of the network to be assessed.

Theorem 1. In a medium to high average SNR regime ${ }^{2}(\bar{\gamma} \gg 1)$, the ASER for a conventional OR network with the radio channels modelled as independent and identical Generalized-K radio channels can be derived to give an asymptotic expression as:

$$
\bar{P}_{s e} \approx \begin{cases}\frac{A \Gamma^{N+1}(k-m) a_{1} \beta(m+1, m N)}{C^{m(1+N)} \bar{\gamma}^{m(1+N)}} & \text { for } m<k \\ \frac{A \Gamma^{N+1}(m-k) a_{2} \beta(k+1, k N)}{C^{k(1+N)} \bar{\gamma}^{k(1+N)}} & \text { for } m>k\end{cases}
$$

where $a_{1}=\frac{2^{N} N m^{(m-1) N+m} k^{m(1+N)} \Gamma(m(1+N)+0.5)}{\Gamma^{N+1}(m) \Gamma^{N+1}(k) \sqrt{\pi}}, a_{2}=\frac{2^{N} N k^{(k-1) N+k} m^{k(1+N)} \Gamma(k(1+N)+0.5)}{\Gamma^{N+1}(m) \Gamma^{N+1}(k) \sqrt{\pi}}$ and $\beta($.$) is the beta$ function [15, eq. (6.2.1)]. The remaining parameters have been described above. For a conventional OR network operating over Generalized-K radio channels, the achieved diversity order is $k(N+1)$ when the shadowing is more severe than the fading, and the achieved diversity order is $m(N+1)$ when the fading is more severe than the shadowing.

Proof: The PDF of $\gamma_{i}$ or any random variable which accepts a Maclaurin series expansion around the origin can be approximated by a single term polynomial [21] as $P_{\gamma_{i}}(\chi)=\tau \chi^{t}+O[\chi]$, where $t$ is the first non-zero derivative order of $P_{\gamma_{i}}(\chi)$ for $\chi=0, \tau=P_{\gamma_{i}}^{t}(0) / t$ !, and $O[\chi]$ is a higher order polynomial function of $\chi$. The motivation behind approximating the PDF of $\gamma_{i}$ by a single term polynomial is the fact [21] that for medium to high SNR the conditional symbol error rate, $P_{s e}(e \mid \chi)$, behaves like a delta function at the origin with decreasing amplitude. For a simplified analysis we will represent the PDF of $\gamma_{y}$ and $\gamma_{i}$ in single term polynomial form. Representing $P_{\gamma_{y}}(\rho)$ in single term polynomial form requires

\footnotetext{
${ }^{2}$ We are using the fact that for independent and identically distributed Generalized- $K$ random variables, $m_{y}=m, k_{y}=k$, and $\bar{\gamma}_{y}=\bar{\gamma}$.
} 
the first non-zero derivative order of $P_{\gamma_{y}}(\rho)$ at $\rho=0$ and the related coefficient for different combinations of the values of $m$ and $k$ to be determined; and then generalising the findings, where this procedure has been adopted from reference [22]. $P_{\gamma_{y}}(\rho)$ is obtained as shown below:

$$
P_{\gamma_{y}}(\rho) \approx\left\{\begin{array}{c}
\frac{k \phi_{1}}{\bar{\gamma}^{k}} \rho^{k-1} \text { for } m>k \\
\frac{m \phi_{2}}{\bar{\gamma}^{m}} \rho^{m-1} \text { for } m<k
\end{array}\right.
$$

where $\phi_{1}=\frac{m^{k} \Gamma(m-k)}{k^{1-k} \Gamma(m) \Gamma(k)}$ and $\phi_{2}=\frac{k^{m} \Gamma(k-m)}{m^{1-m} \Gamma(m) \Gamma(k)}$. The CDF $F_{\gamma_{i}}(\delta)$ is obtained by exploiting the independence of fading gains in the dual-hop relay paths and solving [20, eq. (6.81)] as:

$$
F_{\gamma_{i}}(\delta) \approx 1-\left[1-\frac{m^{k} \Gamma(m-k) \delta^{k}}{\bar{\gamma}^{k} k^{1-k} \Gamma(m) \Gamma(k)}\right]^{2} \text { for } m>k
$$

The PDF $P_{\gamma_{i}}(\delta)$ is derived by taking the derivative of $F_{\gamma_{i}}(\delta)$ :

$$
P_{\gamma_{i}}(\delta) \approx \frac{2 \Gamma(m-k)\left[1-\frac{m^{k} \Gamma(m-k) \delta^{k}}{\bar{\gamma}^{k} k^{1-k} \Gamma(m) \Gamma(k)}\right]}{\delta^{1-k}(k m / \bar{\gamma})^{-k} \Gamma(m) \Gamma(k)} \text { for } m>k .
$$

In order to obtain $P_{\gamma_{i}}(\delta)$ in single term polynomial form, we obtain the first non-zero derivative order of $P_{\gamma_{i}}(\delta)$ at $\delta=0$ and the related coefficient for different combinations of the values of $m$ and $k$ and then generalise the results:

$$
P_{\gamma_{i}}(\delta) \approx \frac{(k m)^{k} 2 \Gamma(m-k) \delta^{k-1}}{\bar{\gamma}^{k} \Gamma(m) \Gamma(k)} \text { for } m>k
$$

By exploiting the fact that the fading gains are independent in the OR paths and using the order statistic theorem [[24], eq. (6.78)], the $\operatorname{CDF} F_{\gamma_{t o t}}(\delta)$ of $\gamma_{t o t}=\max _{i \in\{1, \ldots, N\}}\left(\gamma_{i}\right)$ is derived as:

$$
F_{\gamma_{t o t}}(\delta) \approx \frac{(k)^{(k-1) N} m^{k N} \Gamma^{N}(m-k) 2^{N} \delta^{k N}}{\bar{\gamma}^{k N} \Gamma^{N}(m) \Gamma^{N}(k)} \text { for } m>k .
$$

The PDF $P_{\gamma_{t o t}}(\delta)$ is obtained from the derivative of $F_{\gamma_{t o t}}(\delta)$ :

$$
P_{\gamma_{t o t}}(\delta) \approx \frac{(k)^{(k-1) N+1} m^{k N} \Gamma^{N}(m-k) 2^{N} N \delta^{k N-1}}{\bar{\gamma}^{k N} \Gamma^{N}(m) \Gamma^{N}(k)} \text { for } m>k
$$

In order to find the CDF of $\gamma_{e n d}$, we evaluate the following two-dimensional integral [20, eq. (6.44)]: 


$$
F_{\gamma_{\text {end }}}(\chi)=\int_{\delta=0}^{\chi} \int_{\rho=0}^{\chi-\delta} P_{\gamma_{t o t}}(\delta) P_{\gamma_{s d}}(\rho) d \rho d \delta
$$

By solving the integral in (12) for $P_{\gamma_{t o t}}(\delta)$ in (11), $P_{\gamma_{s d}}(\rho)$ in (6) and with the help of [21, eq. (3.191.1)], the $\mathrm{CDF} F_{\gamma_{e n d}}(\chi)$ is derived as:

$$
F_{\gamma_{e n d}}(\chi) \approx \frac{a_{3} N \beta(k+1, k N) \chi^{k(1+N)}}{\bar{\gamma}^{k(1+N)}} \text { for } m>k
$$

where $a_{3}=\frac{\Gamma^{N+1}(m-k) m^{k(1+N)}(k)^{(k-1) N+k} 2^{N}}{\Gamma^{N+1}(m) \Gamma^{N+1}(k)}$. By repeating the steps from (6) to (12) for $m<k$, the CDF $F_{\gamma_{e n d}}(\chi)$ is obtained as $^{3}$ :

$$
F_{\gamma_{e n d}}(\chi) \approx \frac{a_{4} N \beta(m+1, m N) \chi^{m(1+N)}}{\bar{\gamma}^{m(1+N)}} \text { for } m<k
$$

where $a_{4}=\frac{\Gamma^{N+1}(k-m)(m)^{(m-1) N+m} k^{m(1+N)} 2^{N}}{\Gamma^{N+1}(m) \Gamma^{N+1}(k)}$. Now, the PDF $P_{\gamma_{e n d}}(\chi)$ is derived through determining the derivative of $F_{\gamma_{e n d}}(\chi)$ as:

$$
P_{\gamma_{\text {end }}}(\chi) \approx\left\{\begin{array}{c}
\frac{a_{3} k(1+N) N \beta(k+1, k N) \chi^{k(1+N)-1}}{\bar{\gamma}^{k(1+N)}} \text { for } m>k \\
\frac{a_{4} m(1+N) N \beta(m+1, m N) \chi^{m(1+N)-1}}{\bar{\gamma}^{m(1+N)}} \text { for } m<k
\end{array} .\right.
$$

Finally, the integral in (4) is evaluated by performing the change of variable, $x=\sqrt{\chi}$, and then using [21, eq. (6.281.1)] to yield the desired asymptotic ASER expression (5).

At high SNR, $\bar{P}_{s e}$ can also be represented as [21]:

$$
\bar{P}_{s e} \approx\left(G_{c} \bar{\gamma}\right)^{-G_{d}}
$$

where $G_{c}$ is the coding gain and $G_{d}$ is the diversity order. Thus, if the ASER is plotted versus the average SNR on a log-log scale then the diversity gain can be represented as the slope of this ASER curve. By comparing $\bar{P}_{s e}$ in (5) with (16) we conclude that $G_{d}$ for a conventional OR network operating over a Generalized- $K$ radio channel is $m(N+1)$ for $m<k$ and $G_{d}$ is $k(N+1)$ for $m>k$.

Theorem 2. In a medium to high average SNR regime ${ }^{4}(\bar{\gamma} \gg 1)$, the ASER for a conventional OR network operating over independent and identical Nakagami-m radio channels can be derived to yield

\footnotetext{
${ }^{3}$ While our analysis does not support the case when $m=k$, it can approximate it as $m \approx k$ by choosing the relevant case that depends on whether $m$ is slightly larger or slightly smaller than $k$.

${ }^{4}$ We make use of the fact that for independent and identically distributed Nakagami- $m$ random variables, $m_{y}=m$, and $\bar{\gamma}_{y}=\bar{\gamma}$.
} 
the asymptotic expression:

$$
\bar{P}_{s e} \approx \frac{A \beta(m+1, m N) e_{1}}{C^{m(1+N)} \bar{\gamma}^{m(1+N)}}
$$

where $e_{1}=\frac{2^{N} N m^{(m-1) N+m} \Gamma(m(1+N)+0.5)}{\Gamma^{N+1}(m) \sqrt{\pi}}$ and the remaining parameters have been described eariler. Also, for a conventional OR network operating over independent and identical Nakagami-m radio channels, the resulting diversity order is $m(N+1)$.

Proof: In a Nakagami- $m$ fading environment, the CDF $F_{\gamma_{y}}(\xi)$ of the per hop $\operatorname{SNR} \gamma_{y}$, is represented by:

$$
F_{\gamma_{y}}(\xi)=1-\frac{\Gamma\left(m, \frac{m \xi}{\bar{\gamma}}\right)}{\Gamma(m)}
$$

where $\Gamma(.,$.$) is the upper incomplete Gamma function [18, eq. (6.5.3)]. The CDF of \gamma_{i}$ is obtained by exploiting the fact that the fading gains in the dual-hop relay paths are independent and solving [20, eq. (6.81)] as:

$$
F_{\gamma_{i}}(\gamma)=1-\frac{\Gamma^{2}\left(m, \frac{m \gamma}{\bar{\gamma}}\right)}{\Gamma^{2}(m)}
$$

The PDF $P_{\gamma_{i}}($.$) is obtained by taking the derivative of F_{\gamma_{i}}($.$) using [15, eq. (6.5.25)] as follows:$

$$
P_{\gamma_{i}}(\gamma)=\frac{2\left(\frac{m}{\bar{\gamma}}\right)^{m} \exp \left(-\frac{m \gamma}{\bar{\gamma}}\right) \Gamma\left(m, \frac{m \gamma}{\bar{\gamma}}\right)}{\gamma^{1-m} \Gamma^{2}(m)}
$$

The Maclaurin series of $P_{\gamma_{i}}(\gamma)$ is derived by first finding the Maclaurin series of $\Gamma(m, m \gamma / \bar{\gamma})$ and $\exp (-m \gamma / \bar{\gamma})$, and then multiplying these together:

$$
\begin{aligned}
& P_{\gamma_{i}}(\gamma) \approx\left(\frac{m \gamma}{\bar{\gamma}}\right)^{m}\left(\frac{2 / \gamma}{\Gamma^{2}(m)}-\frac{2 m / \bar{\gamma}}{\Gamma^{2}(m)}+\frac{2 m^{2} \gamma}{2 \Gamma^{2}(m) \bar{\gamma}^{2}}\right) \\
& \times\left[\Gamma(m)+\gamma^{m}\left(-\frac{\left(\frac{m}{\bar{\gamma}}\right)^{m}}{m}+\frac{m\left(\frac{m}{\bar{\gamma}}\right)^{m} \gamma}{(1+m) \bar{\gamma}}-\frac{m^{2}\left(\frac{m}{\bar{\gamma}}\right)^{m} \gamma^{2}}{2(2+m) \bar{\gamma}^{2}}\right)\right] .
\end{aligned}
$$

In a Nakagami- $m$ fading environment, the PDF of $\gamma_{s d}$ is given [21] as $P_{\gamma_{s d}}(\psi) \approx \frac{m^{m}}{\bar{\gamma}^{m} \Gamma(m)} \psi^{m-1}$. To obtain the first non-zero derivative order $(t)$ and the related coefficient $(\tau)$ of $P_{\gamma_{i}}($.$) we examine the$ Maclaurin series of $P_{\gamma_{i}}($.$) in (21) for the smallest power term of \gamma$ using different combinations of the 
values of $m$ and $N$. Then generalising these results for $t$ and $\tau, P_{\gamma_{i}}($.$) is obtained as:$

$$
P_{\gamma_{i}}(\gamma) \approx \frac{m^{m} 2}{\bar{\gamma}^{m} \Gamma(m)} \gamma^{m-1}
$$

By exploiting the independence of the radio channel gains in the OR paths and using the order statistic theorem [20, eq. (6.78)], the $\operatorname{CDF} F_{\gamma_{t o t}}(\delta)$ of $\gamma_{t o t}$ is derived as:

$$
F_{\gamma_{t o t}}(\delta) \approx \frac{m^{(m-1) N} 2^{N} \delta^{m N}}{\bar{\gamma}^{m N} \Gamma^{N}(m)}
$$

The PDF $P_{\gamma_{t o t}}(\delta)$ is found by taking the derivative of $F_{\gamma_{t o t}}(\delta)$ in (23) as shown below:

$$
P_{\gamma_{t o t}}(\delta) \approx \frac{m^{(m-1) N+1} 2^{N} N \delta^{m N-1}}{\bar{\gamma}^{m N} \Gamma^{N}(m)}
$$

To find the $\operatorname{CDF} F_{\gamma_{\text {end }}}(\chi)$ of $\gamma_{\text {end }}$, we evaluate the following integral [20, eq. (6.44)]:

$$
F_{\gamma_{\text {end }}}(\chi)=\int_{\gamma=0}^{\chi} \int_{\psi=0}^{\chi-\gamma} P_{\gamma_{\text {tot }}}(\gamma) P_{\gamma_{s d}}(\psi) d \psi d \gamma
$$

By solving (25) using $P_{\gamma_{t o t}}($.$) in (24), P_{\gamma_{s d}}($.$) defined above and with the help of [21, eq. (3.191.1)],$ $F_{\gamma_{\text {end }}}($.$) is derived as follows:$

$$
F_{\gamma_{\text {end }}}(\chi) \approx \frac{m^{(m-1) N+m} e_{2} \chi^{m(1+N)}}{\bar{\gamma}^{m(1+N)}}
$$

where $e_{2}=\frac{2^{N} \beta(m+1, m N) N}{\Gamma^{N+1}(m)}$. Finally, the PDF $P_{\gamma_{e n d}}(\chi)$ is derived by solving the derivative of the CDF $F_{\gamma_{\text {end }}}(\chi)$ :

$$
P_{\gamma_{\text {end }}}(\chi) \approx \frac{m^{(m-1) N+m+1}(1+N) e_{2} \chi^{m(1+N)-1}}{\bar{\gamma}^{m(1+N)}} .
$$

Finally, the ASER is derived by substituting (27) in (4) and then performing the change of variable, $x=\sqrt{\chi}$, and using [21, eq. (6.281.1)] to yield the expression for ASER (17). Note that, although Theorem 2 has been derived from fundamentals, the result for Nakagami- $m$ channels can also be obtained from Theorem 1 by applying the limit to expression (5) when $k \rightarrow \infty$, which further validates our approach.

\section{B. Outage Probability}


Outage probability $\left(P_{\text {out }}\right)$ is a standard performance criterion for wireless communication systems operating over radio channels and is defined as the probability that the received SNR of a wireless communication system falls below a certain specified threshold SNR $\left(\gamma_{t h}\right)$. For a conventional OR network, outage probability can be represented as:

$$
P_{\text {out }}=\operatorname{Pr}\left[\gamma_{\text {end }}<\gamma_{t h}\right]=F_{\gamma_{\text {end }}}\left(\gamma_{t h}\right)
$$

Now, substituting $F_{\gamma_{\text {end }}}(\chi)$ from (13) and (14) into (28), and noting that $\chi=\gamma_{t h}$, we obtain the asymptotic expression for the outage probability of a conventional OR network operating over Generalized$K$ radio channels as:

$$
P_{\text {out }} \approx\left\{\begin{array}{c}
\frac{2^{N} \Gamma^{N+1}(m-k) m^{k(1+N)} N \beta(k+1, k N)(k)^{(k-1) N+k} \gamma_{t h}^{k(1+N)}}{\bar{\gamma}^{k(1+N)} \Gamma^{N+1}(m) \Gamma^{N+1}(k)} \text { for } m>k \\
\frac{2^{N} \Gamma^{N+1}(k-m) k^{m(1+N)} N \beta(m+1, m N)(m)^{(m-1) N+m} \gamma_{t h}^{m(1+N)}}{\bar{\gamma}^{m(1+N)} \Gamma^{N+1}(m) \Gamma^{N+1}(k)} \text { for } m<k
\end{array} .\right.
$$

Similarly, substituting (26) in (28), the asymptotic expression for the outage probability of a conventional OR network operating over Nakagami- $m$ radio channels is derived as:

$$
P_{\text {out }} \approx \frac{2^{N} m^{(m-1) N+m} \beta(m+1, m N) N \gamma_{t h}^{m(1+N)}}{\bar{\gamma}^{m(1+N)} \Gamma^{N+1}(m)}
$$

IV. Performance Analysis of Opportunistic Hybrid-Arq Incremental Relaying

\section{NETWORKS}

\section{Average Symbol Error Rate}

In OHIR, the source node transmits the signal, which is received by the destination and relays. The destination evaluates the signal, and if necessary, requests the relays to retransmit the signal they received from the source. The retransmitted signal is then combined at the destination using Maximum Ratio Combining (MRC). As the destination evaluates a signal based on the instantaneous SNR, the ASER for an AF OHIR network [9] can be written as:

$$
\bar{P}_{s e}=\operatorname{Pr}\left(\gamma_{s d} \leq \gamma_{t h}\right) \bar{P}_{s e_{\text {combo }}}+\left(1-\operatorname{Pr}\left(\gamma_{s d} \leq \gamma_{t h}\right)\right) \bar{P}_{\text {se } e_{\text {direct }}}
$$

where $\bar{P}_{s e_{\text {combo }}}$ is the average probability that an error occurs at the destination in the case of combined signal transmission from the source and the opportunistic Hybrid-ARQ incremental cooperative relay. 
$\bar{P}_{\text {se }_{\text {direct }}}$ is the ASER for direct transmission. For direct transmission only, $\gamma_{t h}$ is equal to 0 ; for conventional OR, $\gamma_{t h}$ is equal to $\infty$. The ASER with direct transmission only can be written as:

$$
\bar{P}_{\text {se direct }}=\int_{0}^{\infty} P_{\gamma_{s d}}\left(\chi \mid\left(\gamma_{s d}>\gamma_{t h}\right)\right) P_{s e}(e \mid \chi) d \chi
$$

Theorem 3. In a medium to high average SNR range $(\bar{\gamma} \gg 1)$, the ASER for an AF OHIR network operating over independent and identical Generalized-K radio channels can be derived in an asymptotic expression as shown below:

$\bar{P}_{s e} \approx\left\{\begin{array}{l}\left(\frac{A \phi_{1}}{\bar{\gamma}^{k}}\right)\left(\frac{\Gamma\left(k+0.5, C \gamma_{t h}\right)}{\sqrt{\pi} C^{k}}-\frac{\operatorname{erfc}\left(\sqrt{\mathrm{C} \gamma_{\mathrm{th}}}\right)}{\gamma_{t h}^{-k}}\right)+\left(\frac{A \phi_{1}\left(\frac{\gamma_{t h}}{\bar{\gamma}}\right)^{k}}{\bar{\gamma}^{k(1+N)}}\right)\left(\frac{\operatorname{erfc}\left(\sqrt{C \gamma_{t h}}\right)}{\left(r_{4} r_{5}\right)^{-1}}+r_{4} r_{6}+N a_{3} \sum_{l=0}^{k} u_{l} \gamma_{t h}^{w_{l}} q_{l}\right) \text { for } m>k \\ \left(\frac{A \phi_{2}}{\bar{\gamma}^{m}}\right)\left(\frac{\Gamma\left(m+0.5, C \gamma_{t h}\right)}{\sqrt{\pi} C^{m}}-\frac{\operatorname{erfc}\left(\sqrt{C \gamma_{t h}}\right)}{\gamma_{t h}^{-m}}\right)+\left(\frac{A \phi_{2}\left(\frac{\gamma_{t h}}{\bar{\gamma}}\right)^{m}}{\bar{\gamma}^{m(1+N)}}\right)\left(\frac{\operatorname{ercc}\left(\sqrt{C \gamma_{t h}}\right)}{\left(r_{1} r_{2}\right)^{-1}}+r_{1} r_{3}+N a_{4} \sum_{l=0}^{m} c_{l} \gamma_{t h}^{p_{l}} q_{l}\right) \text { for } m<k\end{array}\right.$

where $p_{l}=m(1+N)-l, w_{l}=k(1+N)-l, u_{l}=\left(\begin{array}{c}k \\ l\end{array}\right) \frac{(-1)^{k-l}}{(k(1+N)-l)}, c_{l}=\left(\begin{array}{c}m \\ l\end{array}\right) \frac{(-1)^{m-l}}{(m(1+N)-l)}, q_{l}=\frac{\Gamma\left(0.5+l, C \gamma_{t h}\right)}{\sqrt{\pi} C^{l}}-$ $\operatorname{erfc}\left(\sqrt{C \gamma_{t h}}\right) \gamma_{t h}^{l}, r_{1}=\frac{a_{4} \beta(m N+1, m)}{\sqrt{\pi} C^{m(1+N)}}, r_{2}=\left(C \gamma_{t h}\right)^{m(1+N)} \sqrt{\pi}, r_{3}=\Gamma(0.5+m(1+N))-\Gamma\left(0.5+m(1+N), C \gamma_{t h}\right), r_{4}=$ $\frac{a_{3} \beta(k N+1, k)}{\sqrt{\pi} C^{k(1+N)}}, r_{5}=\left(C \gamma_{t h}\right)^{k(1+N)} \sqrt{\pi}, r_{6}=\Gamma(0.5+k(1+N))-\Gamma\left(0.5+k(1+N), C \gamma_{t h}\right)$. All other parameters have been defined previously.

Proof: The probability that the instantaneous SNR of the direct path falls below the threshold SNR is derived as follows:

$$
\operatorname{Pr}\left(\gamma_{s d} \leq \gamma_{t h}\right)=F_{\gamma_{s d}}\left(\gamma_{t h}\right) \approx \frac{\phi_{1} \gamma_{t h}^{k}}{\bar{\gamma}^{k}} \text { for } m>k
$$

The conditional CDF that the instantaneous SNR of the direct path is greater than the threshold SNR is derived as:

$$
F_{\gamma_{s d}}\left(\chi \mid\left(\gamma_{s d}>\gamma_{t h}\right)\right)= \begin{cases}0 & \text { for } \chi \leq \gamma_{t h} \\ \frac{F_{\gamma_{s d}}(\gamma)-F_{\gamma_{s d}}\left(\gamma_{t h}\right)}{1-F_{\gamma_{s d}}\left(\gamma_{t h}\right)} & \text { for } \chi>\gamma_{t h}\end{cases}
$$

The conditional PDF that the instantaneous SNR of the direct path is greater than the threshold SNR is derived by taking the derivative of the conditional $\operatorname{CDF} F_{\gamma_{s d}}\left(\chi \mid\left(\gamma_{s d}>\gamma_{t h}\right)\right)$ :

$$
P_{\gamma_{s d}}\left(\chi \mid\left(\gamma_{s d}>\gamma_{t h}\right)\right)=\left\{\begin{array}{cr}
0 & \text { for } \chi \leq \gamma_{t h} \\
\left(\frac{k \phi_{1}}{\bar{\gamma}^{k}-\phi_{1} \gamma_{t h}^{k}}\right) \chi^{k-1} \text { for } \chi>\gamma_{t h} & \text { for } m>k
\end{array}\right.
$$


Finally, the ASER of OHIR network with direct transmission only is evaluated by first substituting $P_{\gamma_{s d}}\left(\chi \mid\left(\gamma_{s d}>\gamma_{t h}\right)\right)(36)$ in (32) and then solving the integral:

$$
\bar{P}_{\text {se } e_{\text {direct }}}=\left(\frac{A \phi_{1}}{\bar{\gamma}^{k}-\phi_{1} \gamma_{t h}^{k}}\right)\left(\frac{\Gamma\left(k+0.5, C \gamma_{t h}\right)}{\sqrt{\pi} C^{k}}-\gamma_{t h}^{k} \operatorname{erfc}\left(\sqrt{\mathrm{C} \gamma_{\mathrm{th}}}\right)\right) \text { for } m>k
$$

By using the same procedure from (34) to (37), the ASER $\bar{P}_{\text {sedirect }_{\text {din }}}$ for $m<k$ is derived as:

$$
\bar{P}_{\text {se direct }}=\left(\frac{A \phi_{2}}{\bar{\gamma}^{m}-\phi_{2} \gamma_{t h}^{m}}\right)\left(\frac{\Gamma\left(m+0.5, C \gamma_{t h}\right)}{\sqrt{\pi} C^{m}}-\gamma_{t h}^{m} \operatorname{erfc}\left(\sqrt{\mathrm{C} \gamma_{\text {th }}}\right)\right) \text { for } m<k
$$

The combined ASER of OHIR network can be written as:

$$
\bar{P}_{s_{\text {combo }}}=\int_{0}^{\infty} P_{\gamma_{e n d}}\left(\chi \mid\left(\gamma_{s d} \leq \gamma_{t h}\right)\right) P_{s e}(e \mid \chi)
$$

The conditional CDF of the instantaneous SNR of the combined signal (from both the direct path and OHIR), given that the instantaneous SNR of the direct path falls below the threshold SNR, can be derived as:

$$
F_{\gamma_{e n d}}\left(\chi \mid\left(\gamma_{s d} \leq \gamma_{t h}\right)\right)=\left\{\begin{array}{l}
\int_{\rho=0}^{\chi} P_{\gamma_{s d}}(\rho) F_{\gamma_{t o t}}(\chi-\rho) \mathrm{d} \rho \text { for } \chi \leq \gamma_{t h} \\
\int_{\rho=0}^{\gamma_{t h}} P_{\gamma_{t o t}}(\rho) F_{\gamma_{s d}}(\chi-\rho) d \rho \text { for } \chi>\gamma_{t h}
\end{array}\right.
$$

By solving the integral in (40) for $F_{\gamma_{s d}}($.$) in (34), P_{\gamma_{t o t}}($.$) in (11), F_{\gamma_{t o t}}($.$) in (10), P_{\gamma_{s d}}($.$) in (6) and$ with the help of [21, eq. (3.191.1)], the conditional $\operatorname{CDF} F_{\gamma_{\text {end }}}\left(\chi \mid\left(\gamma_{s d} \leq \gamma_{t h}\right)\right)$ is derived as:

$$
F_{\gamma_{\text {end }}}\left(\chi \mid\left(\gamma_{s d} \leq \gamma_{t h}\right)\right)=\left\{\begin{array}{c}
\left(\frac{a_{3} \beta(k N+1, k)}{\bar{\gamma}^{k(1+N)}}\right) \chi^{k(1+N)} \quad \text { for } \chi \leq \gamma_{t h} \\
\left(\frac{N a_{3}}{\bar{\gamma}^{k(1+N)}}\right) \sum_{l=0}^{k} u_{l} \gamma_{t h}^{k(1+N)-l} \chi^{l} \text { for } \chi>\gamma_{t h}
\end{array} \text { for } m>k\right.
$$

The conditional PDF of the instantaneous SNR of the combined signal, given that the instantaneous SNR of the direct path falls below the threshold SNR, is derived by taking the derivative of the conditional $\operatorname{CDF} F_{\gamma_{e n d}}\left(\chi \mid\left(\gamma_{s d} \leq \gamma_{t h}\right)\right)$ :

$$
P_{\gamma_{e n d}}\left(\chi \mid\left(\gamma_{s d} \leq \gamma_{t h}\right)\right)=\left\{\begin{array}{ll}
\frac{a_{3}(1+N) k \beta(k N+1, k) \chi^{k(1+N)-1}}{\bar{\gamma}^{k(1+N)}} & \text { for } \chi \leq \gamma_{t h} \\
\frac{N a_{3} \sum_{l=0}^{k} l u_{l} \gamma_{t h}^{(1+N)-l} \chi^{l-1}}{\bar{\gamma}^{k(1+N)}} & \text { for } \chi>\gamma_{t h}
\end{array} \text { for } m>k\right.
$$


By using the same procedure from (40) to (42), the PDF $P_{\gamma_{e n d}}\left(\chi \mid\left(\gamma_{s d} \leq \gamma_{t h}\right)\right)$ for $m<k$ is derived as:

$$
P_{\gamma_{e n d}}\left(\chi \mid\left(\gamma_{s d} \leq \gamma_{t h}\right)\right)=\left\{\begin{array}{ll}
\frac{a_{4}(1+N) m \beta(m N+1, m) \chi^{m(1+N)-1}}{\bar{\gamma}^{m(1+N)}} & \text { for } \chi \leq \gamma_{t h} \\
\frac{N a_{4} \sum_{l=0}^{m} l c_{l} \gamma_{t h}^{m(1+N)-l} \chi^{l-1}}{\bar{\gamma}^{m(1+N)}} & \text { for } \chi>\gamma_{t h}
\end{array} \text { for } m<k\right.
$$

Finally, the ASER in the case of combined transmission from both OHIR and the direct path is derived by first substituting $P_{\gamma_{e n d}}($.$) (42) for m>k$ in (39) and $P_{\gamma_{e n d}}($.$) (43) for m<k$ in (39) and then solving the integral as shown below:

$$
\bar{P}_{s e_{\text {combo }}}=\left\{\begin{array}{l}
\frac{A}{\bar{\gamma}^{(1+N)}}\left(r_{4} r_{5} \operatorname{erfc}\left(\sqrt{C \gamma_{t h}}\right)+r_{4} r_{6}+N a_{3} \sum_{l=0}^{k} u_{l} \gamma_{t h}^{w_{l}} q_{l}\right) \text { for } m>k \\
\frac{A}{\bar{\gamma}^{m(1+N)}}\left(r_{1} r_{2} \operatorname{erfc}\left(\sqrt{C \gamma_{t h}}\right)+r_{1} r_{3}+N a_{4} \sum_{l=0}^{m} c_{l} \gamma_{t h}^{p_{l}} q_{l}\right) \text { for } m<k
\end{array} .\right.
$$

where all parameters have been defined previously.

Now, substituting (34), (37) and (44) in (31) yields the desired comprehensive asymptotic ASER expression for an OHIR network operating over Generalized- $K$ radio channels and completes the proof.

Theorem 4. In a medium to high average SNR range $(\bar{\gamma} \gg 1)$, the ASER for an AF OHIR network operating over independent and identical Nakagami-m radio channels can be derived to yield an asymptotic expression as shown below:

$\bar{P}_{s e}=\left(\frac{A \Omega}{\bar{\gamma}^{m}}\right)\left(\frac{\Gamma\left(m+0.5, C \gamma_{t h}\right)}{\sqrt{\pi} C^{m}}-\frac{\operatorname{erfc}\left(\sqrt{\mathrm{C} \gamma_{\mathrm{th}}}\right)}{\gamma_{t h}^{-m}}\right)+\left(\frac{A \Omega\left(\frac{\gamma_{t h}}{\bar{\gamma}}\right)^{m}}{\bar{\gamma}^{m(1+N)}}\right)\left(\frac{\operatorname{erfc}\left(\sqrt{C \gamma_{t h}}\right)}{\left(b_{2} r_{2}\right)^{-1}}+b_{2} r_{3}+N b_{1} \sum_{l=0}^{m} c_{l} \gamma_{t h}^{p_{l}} q_{l}\right)$

where $\Omega=\frac{m^{m-1}}{\Gamma(m)}, b_{1}=\frac{2^{N} m^{(m-1) N+m}}{\Gamma^{N+1}(m)}, b_{2}=\frac{b_{1} \beta(m N+1, m)}{\sqrt{\pi} C^{m(1+N)}}$ and all other parameters have been defined previously.

Proof: In a Nakagami- $m$ fading environment, the probability that the instantaneous SNR of the direct path falls below the threshold SNR is:

$$
\operatorname{Pr}\left(\gamma_{s d} \leq \gamma_{t h}\right)=F_{\gamma_{s d}}\left(\gamma_{t h}\right) \approx \frac{\Omega \gamma_{t h}^{m}}{\bar{\gamma}^{m}}
$$

In a Nakagami- $m$ fading environment, the conditional CDF that the instantaneous SNR of the direct path is greater than the threshold SNR is:

$$
F_{\gamma_{s d}}\left(\chi \mid\left(\gamma_{s d}>\gamma_{t h}\right)\right)= \begin{cases}0 & \text { for } \chi \leq \gamma_{t h} \\ \frac{F_{\gamma_{s d}}(\gamma)-F_{\gamma_{s d}}\left(\gamma_{t h}\right)}{1-F_{\gamma_{s d}}\left(\gamma_{t h}\right)} & \text { for } \chi>\gamma_{t h}\end{cases}
$$


In a Nakagami- $m$ fading environment, the conditional PDF for when the instantaneous SNR of the direct path is greater than the threshold SNR is derived by taking the derivative of the conditional CDF $F_{\gamma_{s d}}\left(\chi \mid\left(\gamma_{s d}>\gamma_{t h}\right)\right)$ :

$$
P_{\gamma_{s d}}\left(\chi \mid\left(\gamma_{s d}>\gamma_{t h}\right)\right)=\left\{\begin{array}{cc}
0 & \text { for } \chi \leq \gamma_{t h} \\
\left(\frac{m^{m}}{\Gamma(m)\left(\bar{\gamma}^{m}-\Omega \gamma_{t h}^{m}\right)}\right) \chi^{m-1} & \text { for } \chi>\gamma_{t h}
\end{array} .\right.
$$

Finally, the ASER of OHIR network with direct transmission only is evaluated by first substituting $P_{\gamma_{s d}}\left(\chi \mid\left(\gamma_{s d}>\gamma_{t h}\right)\right)(48)$ in (32) and then solving the integral:

$$
\bar{P}_{\text {se }_{\text {direct }}}=\left(\frac{A \Omega}{\bar{\gamma}^{m}-\Omega \gamma_{t h}^{m}}\right)\left(\frac{\Gamma\left(m+0.5, C \gamma_{t h}\right)}{\sqrt{\pi} C^{m}}-\gamma_{t h}^{m} \operatorname{erfc}\left(\sqrt{\mathrm{C} \gamma_{\mathrm{th}}}\right)\right) .
$$

By solving the integral in (40) for $P_{\gamma_{t o t}}($.$) in (24), F_{\gamma_{s d}}($.$) in (46), P_{\gamma_{s d}}(\rho)$ defined in Theorem 2 and $F_{\gamma_{t o t}}($.$) in (23), and with the help of [21, eq. (3.191.1)], the conditional CDF F_{\gamma_{\text {end }}}\left(\chi \mid\left(\gamma_{s d} \leq \gamma_{t h}\right)\right)$ is derived as:

$$
F_{\gamma_{e n d}}\left(\chi \mid\left(\gamma_{s d} \leq \gamma_{t h}\right)\right)=\left\{\begin{array}{l}
\frac{b_{1} \chi^{m(1+N)} \beta(m N+1, m)}{\bar{\gamma}^{m(1+N)}} \text { for } \chi \leq \gamma_{t h} \\
\frac{N b_{1} \sum_{l=0}^{m} c_{l} \gamma_{t h}^{m(1+N)-l} \chi^{l}}{\bar{\gamma}^{m(1+N)}} \text { for } \chi>\gamma_{t h}
\end{array}\right.
$$

The conditional PDF of the instantaneous SNR of the combined signal given that the instantaneous SNR of the direct path falls below the threshold SNR is derived by taking the derivative of the conditional $\operatorname{CDF} F_{\gamma_{e n d}}\left(\chi \mid\left(\gamma_{s d} \leq \gamma_{t h}\right)\right)$ :

$$
P_{\gamma_{\text {end }}}\left(\chi \mid\left(\gamma_{s d} \leq \gamma_{t h}\right)\right)=\left\{\begin{array}{cc}
\frac{b_{1} m(1+N) \beta(m N+1, m) \chi^{m(1+N)-1}}{\bar{\gamma}^{m(1+N)}} & \text { for } \chi \leq \gamma_{t h} \\
\frac{N b_{1} \sum_{l=0}^{m} l c_{l} \gamma_{t h}^{p} \chi^{l-1}}{\bar{\gamma}^{m(1+N)}} & \text { for } \chi>\gamma_{t h}
\end{array}\right.
$$

Finally, the ASER in the case of combined transmission from both OHIR and direct path is evaluated by first substituting $P_{\gamma_{\text {end }}}($.$) (51) in (39) and then solving the integral:$

$$
\bar{P}_{\text {se }_{\text {combo }}}=\frac{A}{\bar{\gamma}^{m(1+N)}}\left(b_{2} r_{2} \operatorname{erfc}\left(\sqrt{C \gamma_{t h}}\right)+b_{2} r_{3}+N b_{1} \sum_{l=0}^{m} c_{l} \gamma_{t h}^{p_{l}} q_{l}\right)
$$

Now, substituting (46), (49) and (52) in (31) yields the desired asymptotic ASER expression (45) for OHIR network operating over Nakagami- $m$ radio channels and completes the proof. 


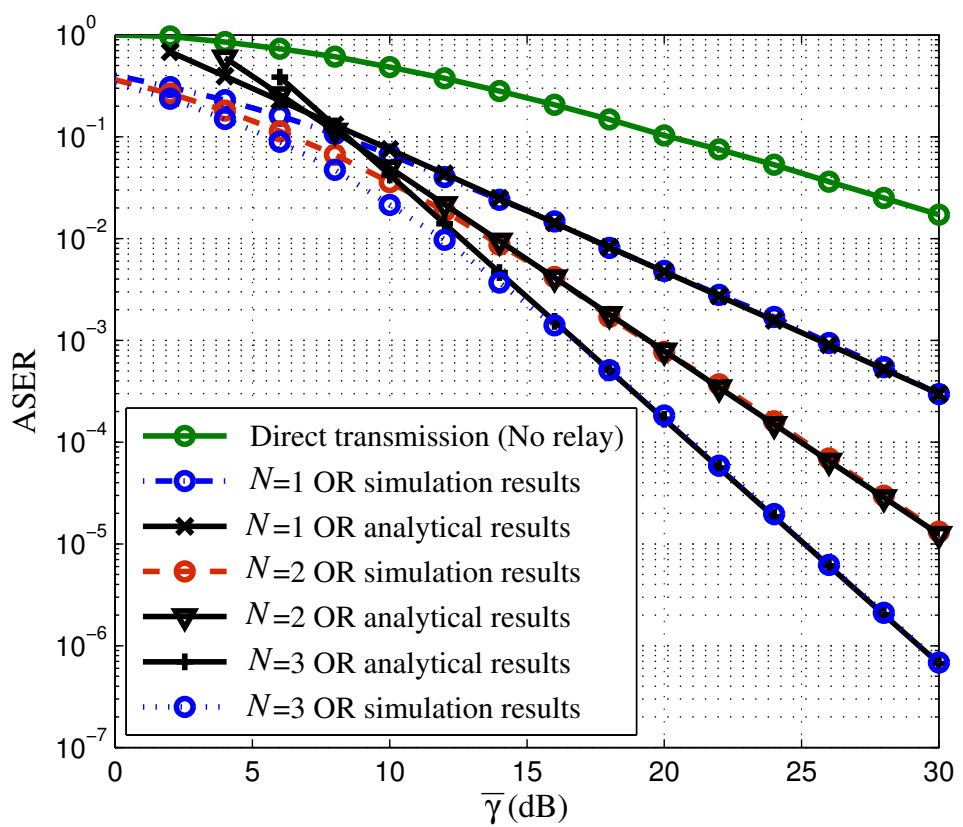

Figure 2. ASER of a conventional OR LTE-Advanced network operating over Generalized- $K$ radio channels. Simulation parameters: $m=0.6, k=38.1, A=1$ and $C=1 / 2$.

\section{NumERicAl AND Simulation Results}

This section presents numerical and simulation results for the outage probability and the ASER of both conventional OR and OHIR networks with the radio channel modelled as Generalized- $K$ and Nakagami- $m$ random processes. We consider two shadowing scenarios that correspond to Loo's model [26], i.e. frequent heavy shadowing and average shadowing. The corresponding standard deviation $(\sigma)$ of the lognormal shadowing is equal to 0.806 and 0.161 [26], for each of the two scenarios. Using the moment matching technique presented in [27], the parameter $k$ of the Generalized- $K$ distribution can be linked to $\sigma$ as $k=1 /\left(\exp \left(\sigma^{2}\right)-1\right)$, which equates to the following values for $k$, i.e. $k=1.1$ and 38.1 respectively. The Technical University of Vienna's LTE downlink link level simulator has been used to obtain the simulation results [28]. The results are all obtained for uncoded QPSK, single antenna link configuration.

In Figs. 2 and 3, numerical and simulation results are plotted for the ASER of a conventional OR LTE-Advanced network as described above and with the radio channel modelled as Generalized- $K$ and Nakagami- $m$ random processes, respectively, and for different numbers of candidate relays $(N)$. The curve for direct transmission is also plotted for comparison. The curves show very good comparison between the numerical and simulation results.

In Figs. 4 and 5, a comparison is provided for the ASER performance of both conventional OR and OHIR networks operating over Generalized- $K$ and Nakagami- $m$ radio channels, respectively, for different 


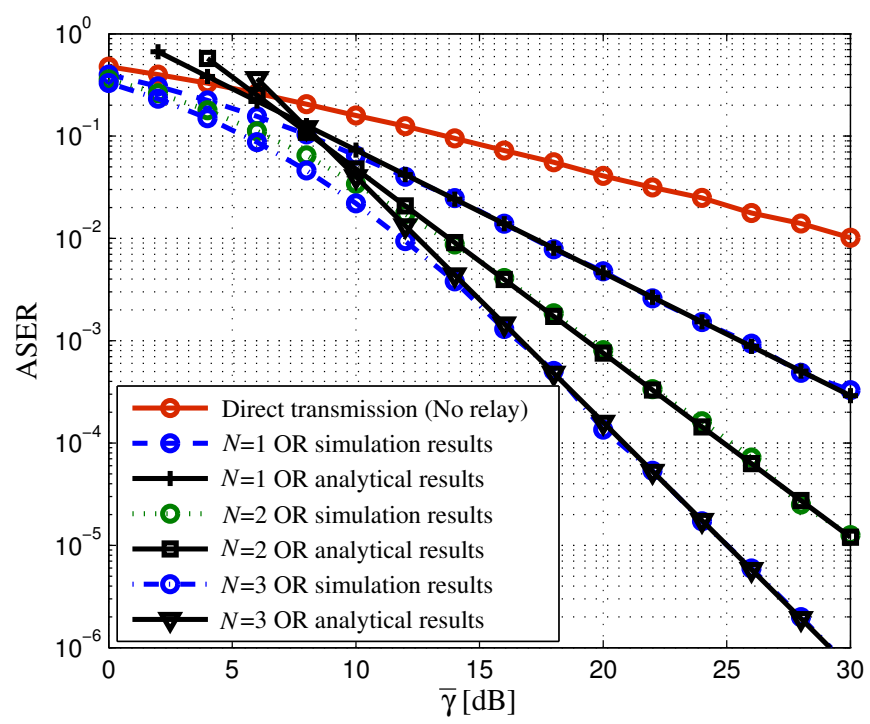

Figure 3. ASER of a conventional OR LTE-Advanced network operating over Nakagami- $m$ radio channels. Simulation parameters: $m=0.6$, $A=1$ and $C=1 / 2$.

values of the threshold SNR $\left(\gamma_{t h}\right)$. A good comparison is shown between the simulation and numerical results, but the curves generated numerically are shown to discontinue for lower values of SNR. This illustrates a limitation of the operational SNR range which the resulting expressions are able to cover.

In Fig. 6, numerical and simulation results are presented for the outage probability $\left(P_{\text {out }}\right)$ of a conventional OR LTE-Advanced network operating over Generalized- $K$ radio channels for different values of the threshold SNR $\left(\gamma_{t h}\right)$ and the number of candidate relays $(N)$. The figure shows good comparison between numerical and simulation results. They illustrate again the limitation of the numerical results at lower SNR values, where the curves are shown to deviate from those resulting from simulations.

From Fig. 2 to Fig. 6, the following observations can be made:

(a) the derived asymptotic results are a good approximation of the outage probability and the ASER for both conventional OR (Figs. 2-3) and OHIR (Figs. 4-5) networks operating over Generalized- $K$ and Nakagami- $m$ radio channels, with the closest approximation being observed for medium to high SNR.

(b) for a conventional OR network operating over Generalized- $K$ radio channels, $P_{\text {out }}$ and $\bar{P}_{\text {se }}$ decrease by a factor of $\bar{\gamma}^{-m(N+1)}$ when fading is more severe than shadowing, and by a factor of $\bar{\gamma}^{-k(N+1)}$ when shadowing is more severe than fading.

(c) the performance of both conventional OR and OHIR networks improves when the number of candidate relays $(N)$ is increased. Fig. 2 compares OR performance with respect to number of 


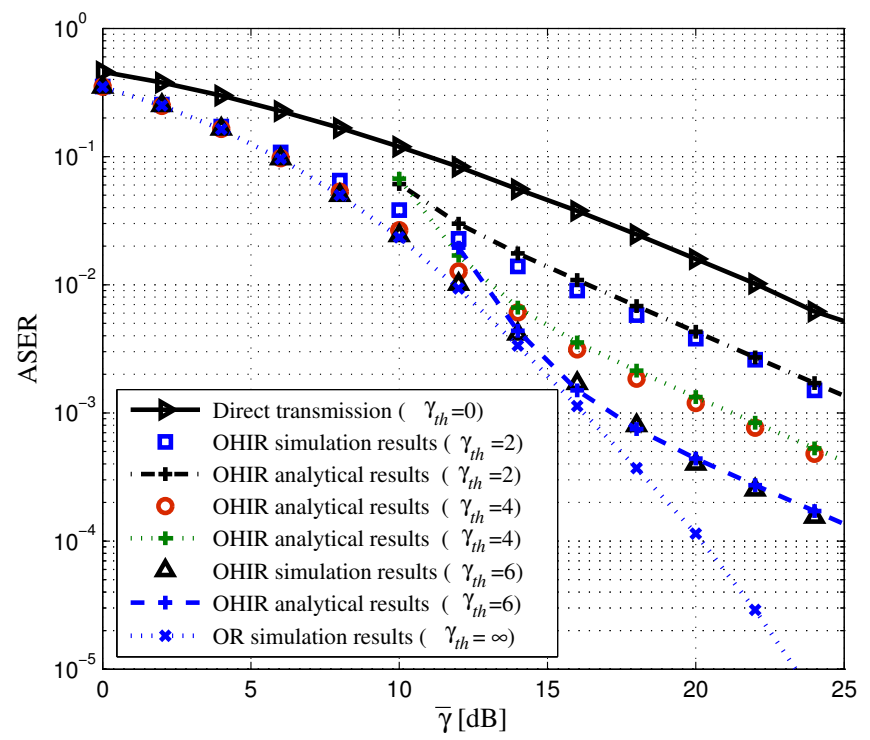

Figure 4. ASER of both conventional OR and OHIR LTE-Advanced networks operating over Generalized- $k$ radio channels. Simulation parameters: $N=2, m=2.5, k=1, A=1$ and $C=1 / 2$.

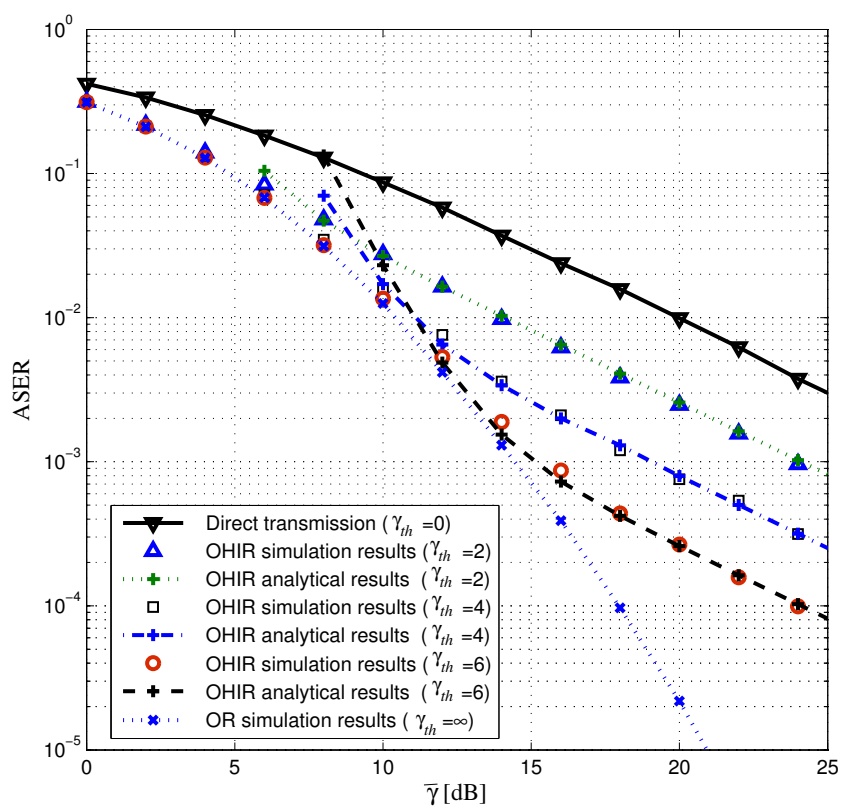

Figure 5. ASER of both conventional OR and OHIR LTE-Advanced networks operating over Nakagami- $m$ radio channels. Simulation parameters: $N=2, m=1, A=1$ and $C=1 / 2$.

relays operating over Generalized- $K$ channels, $(\mathrm{k}=38.1, \mathrm{~m}=0.6)$. At $\mathrm{SNR}=25 \mathrm{~dB}$, the ASER for direct transmission is $2 \times 10^{-2}$, whereas OR achieves $3 \times 10^{-4}, 1 \times 10^{-5,}, 5 \times 10^{-7}$ for $1,2,3$ relay nodes, respectively. Similarly, Fig. 5 compares OHIR performance with respect to number of relays operating over Generalized- $K$ channels $(\mathrm{m}=2.5, \mathrm{k}=1, \mathrm{~A}=1$ and $\mathrm{C}=1 / 2)$. At $\mathrm{SNR}=25 \mathrm{~dB}$, the ASER for direct transmission is $4 \times 10^{-3}$, whereas OHIR achieves $1.5 \times 10^{-3}, 3 \times 10^{-4,}, 1.5 \times 10^{-4}$ 


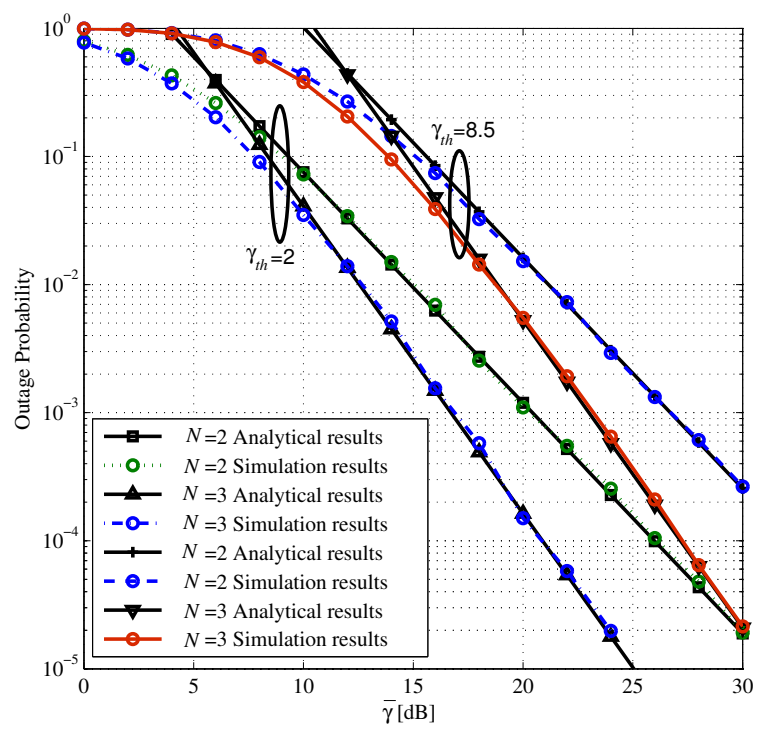

Figure 6. Outage probability $\left(P_{\text {out }}\right)$ of a conventional OR LTE-Advanced network operating over Generalized- $K$ radio channels. Simulation parameters: $m=0.6$ and $k=38.1$.

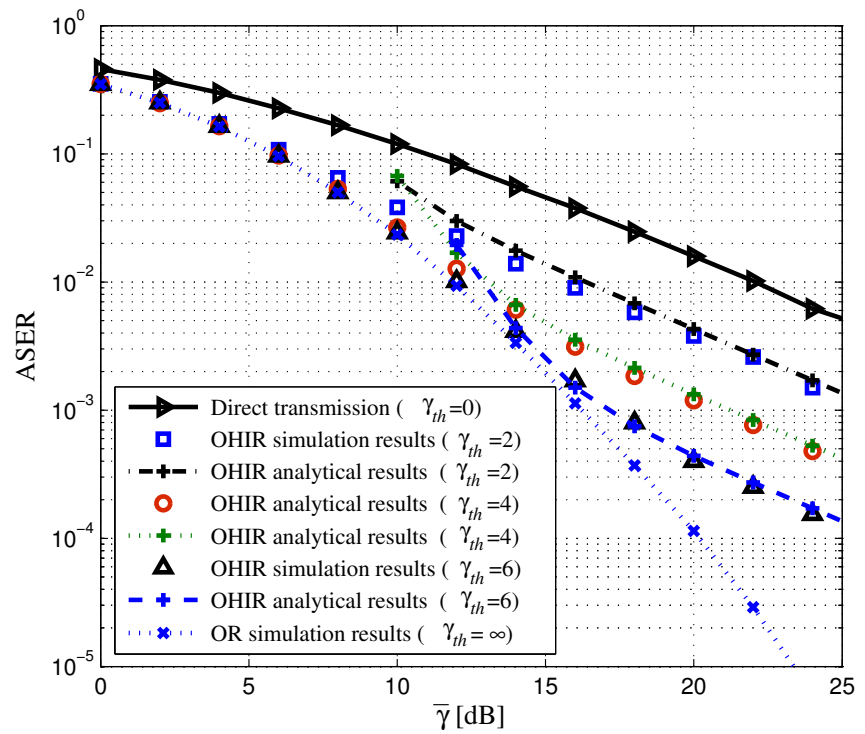

Figure 7. ASER of both conventional OR and OHIR LTE-Advanced networks operating over Generalized- $k$ radio channels. Simulation parameters: $N=2, m=2.5, k=1, A=1$ and $C=1 / 2$.

for $1,2,3$ relay nodes, respectively.

(d) With reference to Figs. 4 and 5, the OHIR network achieves the same ASER performance as that of the conventional OR network at low to medium SNR (for $\bar{\gamma}<\gamma_{t h}$ ). However, at high SNR (for $\bar{\gamma}>\gamma_{t h}$ ) the ASER performance of the OHIR network deviates from the ASER performance of a conventional OR network, but it is much better than the ASER performance of the direct transmission with no relaying. The OR average symbol error rate is lower than that of OHIR. This 
is to be expected as OR relays the signal from the source unconditionally, whereas OHIR relays only if the direct SNR at the destination is below the threshold. Therefore, the SNR of the combined signal in the OR system will always be equal or higher than the combined SNR for OHIR. The higher performance, however, comes at a cost of lower spectral efficiency, as the signal is relayed even if it is not required. The advantage of OHIR is that it is more spectrally efficient than OR while providing a performance better than the baseline, i.e., that for direct transmission from source to destination.

(e) We can see in Figs. 4 and 5 that the ASER performance of the OHIR network improves by increasing the value of the threshold SNR $\left(\gamma_{t h}\right)$. Higher values of threshold make retransmissions more likely because OHIR relays retransmit the signal only if the direct SNR is below the given threshold. The relayed signal is then MRC combined with the direct signal, thus improving an overall ASER.

(f) the outage probability of a conventional OR network degrades with an increase in the threshold SNR. This is due to the fact that as $\gamma_{t h}$ increases, the probability that the SNR of the combined signal (from source and the best relay) is less than $\gamma_{t h}$ will also increase.

\section{CONCLUSIONS}

In this paper, simplified asymptotic expressions for the ASER for both conventional OR and OHIR networks operating over Generalized $K$ and Nakagami- $m$ radio channels have been presented, as well as an expression for the outage probability for OR operating over these channels. The novel expressions are 5, 17, 29, 30, 33 and 45 respectively. The expressions have been validated against results obtained from an established simulator platform. Both theoretical analysis and simulations show that the performance of both conventional OR and OHIR networks improves as the number of candidate relays $(N)$ is increased. The theoretical analysis and simulations confirm that, for a conventional OR network operating over Generalized- $K$ radio channels, the achieved diversity order is $k(N+1)$ when shadowing is more severe than fading, and the achieved diversity order is $m(N+1)$ when fading is more severe than shadowing. The simulation results have also confirmed that OHIR maintains the same quality of service and diversity order as OR while increasing spectral efficiency.

\section{ACKNOWLEDGEMENTS}

The authors would like to thank Professor David Gunton, Dr Alexey Chernov, Dr Faisal Tariq and Dr Eduardo Cano all of the University of Bedfordshire for their helpful comments and suggestions towards 


\section{improving this paper.}

\section{REFERENCES}

[1] M. Peng, Y. Liu, D. Wei, W. Wang, and H. Chen, "Hierarchical cooperative relay based heterogeneous networks," IEEE Wireless Communications Magazine, 2011, 18, (3), pp. 48-56.

[2] S. Sesia, I. Toufik, and M. Baker, "LTE - The UMTS Long Term Evolution: From Theory to Practice" (John Wiley and Sons, 2011, 2nd ed.).

[3] J. N. Laneman, D. N. C. Tse and G. W. Wornell, "Cooperative diversity in wireless networks: Efficient protocols and outage behavior," IEEE Transactions on Information Theory, 2004, 50, (12), pp. 3062-3080.

[4] M. Uysal, ed., "Cooperative Communications for Improved Wireless Network Transmission: Framework for Virtual Antenna Array Applications", IGI Global, July 2009.

[5] J. N. Laneman and G. W. Wornell, "Distributed space-time-coded protocols for exploiting cooperative diversity in wireless networks," IEEE Transactions on Information Theory, 2003, 49, (10), pp. 2415-2425.

[6] A. Bletsas, A. Khisti, D. P. Reed and A. Lippman, "A simple cooperative diversity method based on network path selection," IEEE Journal on Selected Areas in Communications, 2006, 24, (3), pp. 659-672.

[7] A. Bletsas, H. Shin and M. Z. Win, "Cooperative communications with outage-optimal opportunistic relaying," IEEE Transactions on Wireless Communications, 2007, 6, (9), pp. 3450-3460.

[8] B. Zhao and M. C. Valenti, "Practical relay networks: a generalization of hybrid-ARQ," IEEE Journal on Selected Areas in Communications (Special Issue on Wireless Ad Hoc Networks), 2005, 23, (1), pp. 7-18.

[9] S. S. Ikki, M. H. Ahmed, "Performance Analysis of Cooperative Diversity with Incremental-Best-Relay Technique over Rayleigh Fading Channels," IEEE Transactions on Communications, 2011, 59, (8), pp. 2152-2161.

[10] K. S. Hwang, Y. C. Ko, and M. S. Alouini, "Performance analysis of incremental opportunistic relaying over identically and non-identically distributed cooperative paths," IEEE Transactions on Wireless Communications, 2009, 8, (4), pp. 1953-1961.

[11] P. M. Shankar, "Error rates in Generalized shadowed fading channels," Wireless Personal Communications, 2004, 28, (3), pp. $233-238$

[12] P. S. Bithas, N. C. Sagias, P. T. Mathiopoulos, G. K. Karagiannidis, and A. A. Rontogiannis, "On the performance analysis of digital communications over Generalized- $K$ fading channels,” IEEE Communications Letters, 2006, 10, (5), pp. 353-355.

[13] H. Y. Lateef, D. C. Mclernon, and M. Ghogho, "Performance analysis of cooperative communications with opportunistic relaying”, IEEE Signal Processing Advances in Wireless Communications, pp. 1-5, 2010.

[14] M. K. Simon, M. S. Alouini, "Digital Communication over fading channels”, 2nd Ed., Wiley, 2005.

[15] B. Maham and A. Hjorungnes, "Performance analysis of amplify-and-forward opportunistic relaying in rician fading," IEEE Signal Processing Letters, 2009, 16, (8), pp. 643-646.

[16] S. Yadav and P. K. Upadhyay, "Performance analysis of two-way AF relaying systems over cascaded generalized-K fading channels", IEEE National Conference on Communications (NCC), New Delhi, India, 2013, pp. 1-5.

[17] N. Bissias, G. P. Efthymoglou and V. A. Aalo, "Performance analysis of dual-hop relay systems with single relay selection in composite fading channels", International Journal of Electronics and Communications, 2012, 66, (1), pp. 39-44.

[18] M. Abramowitz and I. A. Stegun, "Handbook of Mathematical Functions with Formulas, Graphs, and Mathematical Tables" (Dover, 1972, 9th ed.).

[19] M. O. Hasna and M. S. Alouini, "Outage probability of multihop transmission over Nakagami fading channels," IEEE Communications Letters, 2003, 7, (5), pp. 216-218. 
[20] N. C. Sagias, D. A. Zagos, and G. K. Karagiannidis, "Selection diversity receivers over non-identical Weibull fading channels," IEEE Transactions on Vehicular Technology, 2005, 54, (6), pp. 2146-2151.

[21] Z. Wang and G. B. Giannakis, "A simple and general parameterization quantifying performance in fading channels," IEEE Transactions on Communications, 2003, 51, (8), pp. 1389-1398.

[22] H. Y. Lateef, M. Ghogho and D. C. Mclernon, "On the Performance Analysis of Multi-hop cooperative Relay Networks Over Generalized-K Fading Channels," IEEE Communications Letters, 2011, 15, (9), pp. 968-970.

[23] H. Y. Lateef, M. Ghogho and D.C. McLernon "Performance analysis of multi-hop cooperative relay Networks over generalizedK fading channels", in: 2011 Australian Communications Theory Workshop. 2011.

[24] A. Papoulis, "Probability, Random Variables and Stochastic Processes" (McGraw-Hill, 1991, 3rd ed.).

[25] I. S. Gradshteyn and I. M. Ryzhik, “Table of Integrals, Series and Products” (Academic Press, 2007, 7th ed.).

[26] A. Abdi, W. C. Lau, M. S. Alouni, and M. Kaveh, "A new simple model for land mobile satellite channels: first and second-order statistics," IEEE Transactions on Wireless Communications, 2003, 2, (3), pp. 519-528.

[27] I. M. Kostic, "Analytical approach to performance analysis for channel subject to shadowing and fading," IEE Proceedings Communications, 2005, 152, (6), pp. 821-827.

[28] C. Mehlführer, J. C. Ikuno, M. Simko, S. Schwarz, M. Wrulich and M. Rupp, "The Vienna LTE simulators - Enabling reproducibility in wireless communications research", EURASIP Journal on Advances in Signal Processing, vol. 2011:29, 2011 